\title{
Zur Transformation der ternären quadratischen Formen.
}

\author{
(Von Herrn Paul Bachmann in Breslau.)
}

Herr Hermite hat in diesem Journal Bd. 47, pag. 315 Formeln gegeben, welche alle Transformalionen einer ternären quadratischen Form in sich selbst enthalten. Soviel mir bekannt ist, sind die Bedingungen noch nicht angegeben worden, unter welchen diese Formeln nur diejenigen Transformationen liefern, deren Coefficienten ganze Zahlen sind. Indem ich diese Bedingungen hier mittheilen will, beschränke ich mich auf den besonders einfachen Fall, in welchem folgende Voraussetzungen gelten: Sind $f=\left(\begin{array}{l}a, a^{\prime}, a^{\prime \prime} \\ b, b^{\prime}, b^{\prime \prime}\end{array}\right)$ und $F=\left(\begin{array}{l}A, A^{\prime}, A^{\prime \prime} \\ B, B^{\prime}, B^{\prime \prime}\end{array}\right)$ die ternäre quadratische Form und ihre Adjungirte, so sollen $a, a^{\prime}, a^{\prime \prime}, A, A^{\prime}, A^{\prime \prime}$ ungerade sein; dadurch wird die Determinante $A$ eine ungerade Zahl, welche aus lauter ungleichen Primfactoren bestehen möge, und $b, b^{\prime}, b^{\prime \prime}, B, B^{\prime}, B^{\prime \prime}$ werden gerade Zahlen. Ferner ist $A A^{\prime} A^{\prime \prime} \equiv 3(\bmod .4)$, d. h. unter den drei Zahlen $A, A^{\prime}, A^{\prime \prime}$ ist entweder eine oder jede von der Form $4 n+3$. Von diesen beiden Möglichkeiten schliessen wir im Folgenden die letztere völlig aus. -

1. Die Hermiteschen Formeln enthalten drei Unbestimmte als Elemente, von welchen man bei einer genaueren Betrachtung der Formeln nachweisen kann, dass sie behufs ganzzahliger Transformationen rational zu wählen sind, vorausgesetzt, dass die Determinante $\Delta$ von Null verschieden ist. Bezeichnen hiernach $p, q, q^{\prime}, q^{\prime \prime}$ vier ganze Zahlen ohne gemeinsamen Theiler, so nehmen die Hermiteschen Transformationsgleichungen folgende Gestalt an *):

*) Wo in dieser Abhandlung die Ableitungen von $f$ oder $F$ nach drei gleichnamigen Grössen vorkommen, bedeuten $f$ oder $F$ stets die Werthe, welche sie durch Substitution jener Grössen an Stelle der Unbestimmten annehmen. 


$$
\left\{\begin{array}{c}
{\left[p^{2}-F\left(q, q^{\prime}, q^{\prime \prime}\right)\right] X} \\
=\left(p^{2}+F+2 b^{\prime} p q^{\prime}-2 b^{\prime \prime} p q^{\prime \prime}-q \frac{\partial F}{\partial q}\right) x+\left(2 b p q^{\prime}-2 a^{\prime} p q^{\prime \prime}-q^{\prime} \frac{\partial F}{\partial q}\right) x^{\prime} \\
+\left(2 a^{\prime \prime} p q^{\prime}-2 b p q^{\prime \prime}-q^{\prime \prime} \frac{\partial F}{\partial q}\right) x^{\prime \prime}, \\
{\left[p^{2}-F\left(q, q^{\prime}, q^{\prime \prime}\right)\right] X^{\prime}} \\
=\left(2 a p q^{\prime \prime}-2 b^{\prime} p q-q \frac{\partial F}{\partial q^{\prime}}\right) x+\left(p^{2}+F+2 b^{\prime \prime} p q^{\prime \prime}-2 b p q-q^{\prime} \frac{\partial F}{\partial q^{\prime}}\right) x^{\prime} \\
+\left(2 b^{\prime} p q^{\prime \prime}-2 a^{\prime \prime} p q-q^{\prime \prime} \frac{\partial F}{\partial q^{\prime}}\right) x^{\prime \prime}, \\
{\left[p^{2}-F\left(q, q^{\prime}, q^{\prime \prime}\right)\right] X^{\prime \prime}} \\
=\left(2 b^{\prime \prime} p q-2 a p q^{\prime}-q \frac{\partial F}{\partial q^{\prime \prime}}\right) x+\left(2 a^{\prime} p q-2 b^{\prime \prime} p q^{\prime}-q^{\prime} \frac{\partial F}{\partial q^{\prime \prime}}\right) x^{\prime} \\
+\left(p^{2}+F+2 b p q-2 b^{\prime} p q^{\prime}-q^{\prime \prime} \frac{\partial F}{\partial q^{\prime \prime}}\right) x^{\prime \prime} .
\end{array}\right.
$$

Hierin sind also $p, q, q^{\prime}, q^{\prime \prime}$ so zu wählen, dass die neun Coefficienten zur Rechten, welche wir der Reihe nach durch (1), (2),... (9) bezeichnen wollen, durch $P=p^{2}-F\left(q, q^{\prime}, q^{\prime \prime}\right)$ getheilt, ganze Zahlen ergeben. Untersuchen wir, welche Werthe $P$ haben kann. Aus den Gleichungen aber:

$$
\begin{aligned}
(1)+(5)+(9) & =4 p^{2}-P, \\
a .(1)+b^{\prime \prime} .(4)+b^{\prime} .(7) & =a\left(p^{2}+F\right)-2 \Delta q^{2}, \\
b^{\prime \prime} .(2)+a^{\prime} .(5)+b .(8) & =a^{\prime}\left(p^{2}+F\right)-2 \Delta q^{\prime 2}, \\
b^{\prime} .(3)+b .(6)+a^{\prime \prime} .(9) & =a^{\prime \prime}\left(p^{2}+F\right)-2 \Delta q^{\prime \prime 2}
\end{aligned}
$$

ergeben sich folgende Congruenzbedingungen:

$$
4 p^{2} \equiv 0,2 a p^{2} \equiv 2 \Delta q^{2}, 2 a^{\prime} p^{2} \equiv 2 \Delta q^{\prime 2}, 2 a^{\prime \prime} p^{2} \equiv 2 \Delta q^{\prime \prime 2} \quad(\bmod . P) .
$$

Hieraus schliesst man, dass $4 \Delta p^{2}, 4 \Delta q^{2}, 4 \Delta q^{\prime 2}, 4 \Delta q^{\prime \prime 2}$ und, da $p, q, q^{\prime}, q^{\prime \prime}$ ohne gemeinsamen Theiler sind, auch $4 d$ durch $P$ theilbar sein mưss. Nach den Anfangs gemachten Voraussetzungen hat deshalb $P$ die Form $2^{h} . \delta$, worin $\delta$ ein Theiler von $A$, der auch in $p$ aufgeht, und $h$ eine der Zahlen 0, 1, 2 ist. So finden wir: Damit die Transformation ganzzahlig wird, müssen die ganzen Zahlen $p, q, q^{\prime}, q^{\prime \prime}$ einer Gleichung

$$
\text { (3.) } \quad p^{2}-F\left(q, q^{\prime}, q^{\prime \prime}\right)=2^{h} . \delta
$$

Genüge leisten, während $p \equiv 0(\bmod . \delta)$ ist. Für den Fall $h=2$ lehren ausserdem die Congruenzen (2.), dass $p, q, q^{\prime}, q^{\prime \prime}$ ungerade sein müssen. 
Umgekehrt, wenn für einen oder den andern Divisor $\delta$ von $A$ die Gleichung (3.) in der erwähnten Weise auflösbar ist, so geben die Zahlen $p, q, q^{\prime}, q^{\prime \prime}$ eine ganzzahlige Transformation. - In der That, aus der Gleichung (3.) und wegen $p \equiv 0(\bmod . \delta)$ folgt auch $F\left(q, q^{\prime}, q^{\prime \prime}\right) \equiv=0\left(\bmod . \delta^{\prime}\right)$. Nun besteht die Gleichung *)

$$
\left\{\begin{array}{c}
F\left(x, x^{\prime}, x^{\prime \prime}\right) \cdot F\left(q, q^{\prime}, q^{\prime \prime}\right) \\
=\left(x . \frac{\partial F}{\partial q}+x^{\prime} .1 \frac{\partial F}{\partial q^{\prime}}+x^{\prime \prime} \cdot \frac{\partial F}{\partial q^{\prime \prime}}\right)^{2}-\mathcal{J} \cdot f\left(x^{\prime} q^{\prime \prime}-x^{\prime \prime} q^{\prime}, x^{\prime \prime} q-x q^{\prime \prime}, x q^{\prime}-x^{\prime} q\right)
\end{array}\right.
$$

und ergiebt für alle ganzzahligen $x, x^{\prime}, x^{\prime \prime}$ die Congruenz

und folglich

$$
\boldsymbol{x} \cdot \frac{1}{2} \frac{\partial \boldsymbol{F}}{\partial q}+\boldsymbol{x}^{\prime} \cdot \frac{1}{2} \frac{\partial \boldsymbol{F}}{\partial q^{\prime}}+\boldsymbol{x}^{\prime \prime} \cdot \frac{1}{2} \frac{\partial \boldsymbol{F}}{\partial q^{\prime \prime}} \equiv \mathbf{0} \quad(\bmod \cdot \delta)
$$

(5.) $\quad \frac{1}{2} \frac{\partial F}{\partial q}=0, \quad 2 \frac{\partial F}{\partial q^{\prime}}=0, \quad \frac{c F}{\partial q^{\prime \prime}} \therefore 0 \quad(\bmod \delta)$.

Ist nun zunächst $h=0$ oder $h=1$, so sind nach diesen Congruenzen die Grössen $\frac{\partial F}{\partial q}, \frac{\partial F}{\partial q^{\prime}}, \frac{\partial F}{\partial q^{\prime \prime}}$ durch $P$ theilbar, ferner auch die in $2 p$ multiplicirten Theile der Coefficienten, endlich auch $p^{2}+F=2 p^{2}-P$. - Ist dagegen $h=2$, so werden, wenn $p, q, q^{\prime}, q^{\prime \prime}$ eine Auflösung der Gleichung (3.) in ungeraden Zahlen bedeuten, während die in $b, b^{\prime}, b^{\prime \prime}$ multiplicirten Theile der Coefficienten offenbar durch 4 aufgehen, die andern Theile das Doppelte der Summe zweier ungeraden Zahlen, indem

$\frac{1}{2} \frac{\partial F}{\partial q}=A q+B^{\prime \prime} q^{\prime}+B^{\prime} q^{\prime \prime}, \quad \frac{1}{2} \frac{\partial F}{\partial q^{\prime}}=B^{\prime \prime} q+A^{\prime} q^{\prime}+B q^{\prime \prime}, \quad \frac{1}{2} \frac{\partial F}{\partial q^{\prime \prime}}=B^{\prime} q+B q^{\prime}+A^{\prime \prime} q^{\prime \prime}$ ungerade sind. Also sind die Coefficienten, welche, wie zuvor, durch $\delta$ theilbar sind, auch durch 4 und folglich durch $4 \delta=P$ theilbar.

Es ist jedoch zu bemerken, dass der Fall $h=2$ bei unsern Voraussetzungen nicht in Betracht kommt. Sei zuerst $A \equiv 1$, d. h. $a a^{\prime} a^{\prime \prime} \equiv 3(\bmod .4)$; setzt man $a=2 \alpha+1, a^{\prime}=2 \alpha^{\prime}+1, a^{\prime \prime}=2 \alpha^{\prime \prime}+1$, so ergiebt sich $\alpha+\alpha^{\prime}+\alpha^{\prime \prime} \equiv 1$ (mod.2. Soll andrerseits die Gleichung $p^{2}-F\left(q, q^{\prime}, q^{\prime \prime}\right)=4 \delta$ eine Lösung in ungeraden Zahlen $p, q, q^{\prime}, q^{\prime \prime}$ zulassen, so muss, da $\delta$ ungerade ist, die Congruenz $p^{2}-F\left(q, q^{\prime}, q^{\prime \prime}\right) \equiv 4(\bmod .8$.) bestehen; folglich muss sein

$$
F\left(q, q^{\prime}, q^{\prime \prime}\right) \equiv 5 \quad \text { oder } \quad A+A^{\prime}+A^{\prime \prime}+2\left(B+B^{\prime}+B^{\prime \prime}\right) \equiv 5,
$$

*) S. meine Abh. in diesem Journal Bd. 70 pag. 366. Es sei hier bemerkt, dass in derselben unter $(p, q, r)$ eine eigentlich primitive Form zu verstehen ist, deren Determinante zu $\Delta$ prim ist. 
was nach einfachen Reductionen in $a a^{\prime}+a^{\prime} a^{\prime \prime}+a^{\prime \prime} a \equiv 3$ (mod.8) oder in $(\alpha+1)\left(\alpha^{\prime}+1\right)\left(\alpha^{\prime \prime}+1\right) \equiv \alpha \alpha^{\prime} \alpha^{\prime \prime}+1(\bmod 2)$ übergeht. Die beiden Congruenzen (mod.2) erfordern aber, dass $a, a^{\prime}, a^{\prime \prime}$, folglich auch $A, A^{\prime}, A^{\prime \prime}$ von der Form $4 n+3$ sind. - Ganz ebenso findet man, dass für den Fall $A=3$ (mod.4) die Coefficienten $a, a^{\prime}, a^{\prime \prime}$ von der Form $4 n+1$, also $A, A^{\prime}, A^{\prime \prime}$ wieder von der Form $4 n+3$ sein müssten. Nach unsern Voraussetzungen können daher ungerade Auflösungen der Gleichung (3.) nicht existiren. --

Das erhaltene Resultat lässt sich so aussprechen: Man erhält alle ganzzahligen Transformationen, wenn man für $p, q, q^{\prime}, q^{\prime \prime}$ alle diejenigen Auflösungen der Gleichung $p^{2}-F\left(q, q^{\prime}, q^{\prime \prime}\right)=2^{h} . \delta$ wählt, bei welchen $p=0(\bmod . \delta)$ ist. Diese Gleichung vertritt die Stelle von mehreren, welche erhalten werden, indem für $h$ jeder der Werthe 0,1 , fur $\delta$ jeder (positive oder negative) Divisor von $A$ gesetzt wird. -

2. Herr Hermite hat bereits bemerkt (a. a. 0. pag. 324), dass die quaternäre Form $\xi^{2}-F\left(x, x^{\prime}, x^{\prime \prime}\right)$ die Eigenschaft hat, durch Multiplication sich $z u$ reproduciren. Man beweist dies leicht, indem man dem Producte

$$
\left[\xi^{2}-F\left(x, x^{\prime}, x^{\prime \prime}\right)\right] \cdot\left[\eta^{2}-F\left(y, y^{\prime}, y^{\prime \prime}\right)\right]
$$

mit Hilfe der Formel (4.) die Form giebt

$\left(\xi \eta+x \cdot \frac{1}{2} \frac{\partial F}{\partial y}+x^{\prime} \cdot \frac{\partial}{2} \frac{\partial}{\partial y^{\prime}}+x^{\prime \prime} \cdot \frac{1}{2} \frac{\partial F}{\partial y^{\prime \prime}}\right)^{2}-\left[F^{\prime}\left(\eta x+\xi y, \eta x^{\prime}+\xi y^{\prime}, \eta x^{\prime \prime}+\xi y^{\prime \prime}\right)+\Delta \cdot f\left(s, s^{\prime}, s^{\prime \prime}\right)\right]$, in welcher

$$
\text { (6.) } s=x^{\prime} y^{\prime \prime}-x^{\prime \prime} y^{\prime}, \quad s^{\prime}=x^{\prime \prime} y-x y^{\prime \prime}, \quad s^{\prime \prime}=x y^{\prime}-x^{\prime} y
$$

gesetzt ist, und indem man in dieser das subtraclive Glied gleich

$$
F\left(\eta x+\xi y+t, \eta x^{\prime}+\xi y^{\prime}+t^{\prime}, \eta x^{\prime \prime}+\xi y^{\prime \prime}+t^{\prime \prime}\right)
$$

zu machen sucht dadurch, dass man der Gleichung

$$
\begin{gathered}
(2 \eta x+2 \xi y+t) \cdot \frac{\partial}{2} \frac{\partial F}{\partial t}+\left(2 \eta x^{\prime}+2 \xi y^{\prime}+t^{\prime}\right) \cdot \frac{1}{2} \frac{\partial F}{\partial t^{\prime}}+\left(2 \eta x^{\prime \prime}+2 \xi y^{\prime \prime}+t^{\prime \prime}\right) \cdot \frac{1}{2} \frac{\partial F}{\partial t^{\prime \prime}} \\
=\Delta \cdot f\left(s, s^{\prime}, s^{\prime \prime}\right)
\end{gathered}
$$

Genüge leistet. Setat man nun

so ergiebt sich

$$
t=\frac{1}{2} \frac{\partial f}{\partial s}, \quad t^{\prime}=\frac{1}{2} \frac{\partial f}{\partial s^{\prime}}, \quad t^{\prime \prime}=\frac{1}{2} \frac{\partial f}{\partial s^{\prime \prime}},
$$

$$
\frac{1}{2} \frac{\partial F}{\partial t}=\Delta s, \quad \frac{1}{2} \frac{\partial F}{\partial t^{\prime}}=\Delta s^{\prime}, \quad \frac{1}{2} \frac{\partial F}{\partial t^{\prime \prime}}=\Delta s^{\prime \prime},
$$

und da andrerseits

$$
x s+x^{\prime} s^{\prime}+x^{\prime \prime} s^{\prime \prime}=0, \quad y s+y^{\prime} s^{\prime}+y^{\prime \prime} s^{\prime \prime}=0
$$

ist, so leisten die Werthe (7.) für $t, t^{\prime}, t^{\prime \prime}$ der Forderung Genüge. 
300 Bachmann, zur Transformation der ternären quadratischen Formen.

Es besteht also folgende Gleichung

(8.) $\left[\xi^{2}-F\left(x, x^{\prime}, x^{\prime \prime}\right)\right] \cdot\left[\eta^{2}-F\left(y, y^{\prime}, y^{\prime \prime}\right)\right]=\zeta^{2}-F\left(z, z^{\prime}, z^{\prime \prime}\right)$, wenn man setzt:

$$
\left\{\begin{array}{c}
\zeta=\xi \eta+x \cdot \frac{1}{2} \frac{\partial F}{\partial y}+x^{\prime} \cdot \frac{1}{2} \frac{\partial F}{\partial y^{\prime}}+x^{\prime \prime} \cdot \frac{1}{2} \frac{\partial F}{\partial y^{\prime \prime}} \\
z=\eta x+\xi y+\frac{1}{2} \frac{\partial f}{\partial s}, \quad z^{\prime}=\eta x^{\prime}+\xi y^{\prime}+\frac{1}{2} \frac{\partial f}{\partial s^{\prime}}, \quad z^{\prime \prime}=\eta x^{\prime \prime}+\xi y^{\prime \prime}+\frac{1}{2} \frac{\partial f}{\partial s^{\prime \prime}} .
\end{array}\right.
$$

3. Mit Hülfe dieses Resultates können wir nun zeigen, dass man für ein bestimmtes System $h, \delta$ sämmtliche Auflösungen der Gleichung $p^{2}-F\left(q, q^{\prime}, q^{\prime \prime}\right)=2^{h} . \delta$, bei welchen $p \equiv 0$ (mod. $\delta)$ ist, erhält, indem man irgend eine derselben mit sämmtlichen Auflösungen der Gleichung

$$
\text { (10.) } t^{2}-F\left(u, u^{\prime}, u^{\prime \prime}\right)=1
$$

nach der vorhergehenden Regel zusammensetzt.

In der That, erstens ist offenbar, dass man auf diese Weise nur Auflösungen der Gleichung (3.) von der bezeichneten Art erhält. Denn setzt man so wird

$$
\left[t^{2}-F\left(u, u^{\prime}, u^{\prime \prime}\right)\right] \cdot\left[p^{2}-F\left(q, q^{\prime}, q^{\prime \prime}\right)\right]=p_{1}^{2}-F\left(q_{1}, q_{1}^{\prime}, q_{1}^{\prime \prime}\right),
$$

$$
p_{1}=t p+u \cdot \frac{1}{2} \frac{\partial F}{\partial q}+u^{\prime} \cdot \frac{1}{2} \frac{\partial F}{\partial q^{\prime}}+u^{\prime \prime} \cdot \frac{1}{2} \frac{\partial F}{\partial q^{\prime \prime}} \text {. }
$$

Folglich bilden $p_{1}, q_{1}, q_{1}^{\prime}, q_{1}^{\prime \prime}$ eine Auflösung der Gleichung (3.), bei welcher, da $p \equiv 0(\bmod . \delta)$ angenommen ist, nach den Congruenzen (5.) auch $p_{1} \equiv 0$ $(\bmod . \delta)$ ist.

Zweitens lässt sich zeigen, dass aus je zweien der bezeichneten Auflösungen der Gleichung (3.) eine Auflösung der Gleichung (10.) abgeleitet werden kann. - In der That, wenn $p, q, q^{\prime}, q^{\prime \prime} ; p_{1}, q_{1}, q_{1}^{\prime}, q_{1}^{\prime \prime}$ zwei solche Auflösungen sind, so erhält man

$$
\left[p_{1}^{2}-F\left(q_{1}, q_{1}^{\prime}, q_{1}^{\prime \prime}\right)\right] \cdot\left[p^{2}-F\left(-q,-q^{\prime},-q^{\prime \prime}\right)\right]=p_{2}^{2}-F\left(q_{2}, q_{2}^{\prime}, q_{2}^{\prime \prime}\right)=2^{2 h} . \delta^{2},
$$

wenn man setzt:

$$
\left\{\begin{array}{c}
p_{2}=p p_{1}-\left(q_{1} \cdot \frac{1}{2} \frac{\partial F}{\partial q}+q_{1}^{\prime} \cdot \frac{1}{2} \frac{\partial F}{\partial q^{\prime}}+q_{1}^{\prime \prime} \cdot \frac{1}{2} \frac{\partial F}{\partial q^{\prime \prime}}\right) \\
q_{2}=-p_{1} q+p q_{1}+\frac{1}{2} \frac{\partial f}{\partial\left(q^{\prime} q_{1}^{\prime \prime}-q^{\prime \prime} q_{1}^{\prime}\right)}, \quad q_{2}^{\prime}=-p_{1} q^{\prime}+p q_{1}^{\prime}+\frac{1}{2} \frac{\partial f}{\partial\left(q^{\prime \prime} q_{1}-q q_{1}^{\prime \prime}\right)}, \\
q_{2}^{\prime \prime}=-p_{1} q^{\prime \prime}+p q_{1}^{\prime \prime}+\frac{1}{2} \frac{\partial f}{\partial\left(q q_{1}^{\prime}-q^{\prime} q_{1}\right)} \cdot
\end{array}\right.
$$

Nun findet man, unter Anwendung der Formel (4.), den Voraussetzungen nach leicht folgende Congruenz:

$$
f\left(q^{\prime} q_{1}^{\prime \prime}-q^{\prime \prime} q_{1}^{\prime}, q^{\prime \prime} q_{1}-q q_{1}^{\prime \prime}, q q_{1}^{\prime}-q^{\prime} q_{1}\right) \equiv 0 \quad(\bmod . \delta) \text {. }
$$


Setzt man zur Abkürzung die hierin enthaltenen Argumente gleich $z, z^{\prime}, z^{\prime \prime}$, und

$$
u=q x+q^{\prime} x^{\prime}+q^{\prime \prime} x^{\prime \prime}, \quad u_{1}=q_{1} x+q_{1}^{\prime} x^{\prime}+q_{1}^{\prime \prime} x^{\prime \prime},
$$

so findet man für irgend welche ganze Zahlen $x ; x^{\prime}, x^{\prime \prime}$ die Gleichung

$$
\begin{gathered}
F\left(x^{\prime} z^{\prime \prime}-x^{\prime \prime} z^{\prime}, x^{\prime \prime} z-x z^{\prime \prime}, x z^{\prime}-x^{\prime} z\right) \\
=u_{1}^{2} \cdot F\left(q, q^{\prime}, q^{\prime \prime}\right)-2 u_{1} u\left(q_{1} \cdot \frac{1}{2} \frac{\partial F}{\partial q}+q_{1}^{\prime} \cdot \frac{1}{2} \frac{\partial F}{\partial q^{\prime}}+q_{1}^{\prime \prime} \cdot \frac{1}{2} \frac{\partial F}{\partial q^{\prime \prime}}\right)+u^{2} \cdot F\left(q_{1}, q_{1}^{\prime}, q_{1}^{\prime \prime}\right),
\end{gathered}
$$

also welches auch $x, x^{\prime}, x^{\prime \prime}$ sein mögen, die Congruenz

$$
F\left(x^{\prime} z^{\prime \prime}-x^{\prime \prime} z^{\prime}, x^{\prime \prime} z-x z^{\prime \prime}, x z^{\prime}-x^{\prime} z\right) \equiv 0 \quad(\bmod . \delta) \text {. }
$$

Aus der Gleichung

$$
\begin{gathered}
f\left(x, x^{\prime}, x^{\prime \prime}\right) \cdot f\left(z, z^{\prime}, z^{\prime \prime}\right) \\
=\left(x \cdot \frac{1}{2} \frac{\partial f}{\partial z}+x^{\prime} \cdot \frac{1}{2} \frac{\partial f}{\partial z^{\prime}}+x^{\prime \prime} \cdot \frac{1}{2} \frac{\partial f}{\partial z^{\prime \prime}}\right)^{2}-F\left(x^{\prime} z^{\prime \prime}-x^{\prime \prime} z^{\prime}, x^{\prime \prime} z-x z^{\prime \prime}, x z^{\prime}-x^{\prime} z\right)
\end{gathered}
$$

folgt also für alle ganzzahligen $x, x^{\prime}, x^{\prime \prime}$ die Congruenz

$$
x \cdot \frac{1}{2} \frac{\partial f}{\partial z}+x^{\prime} \cdot \frac{1}{2} \frac{\partial f}{\partial z^{\prime}}+x^{\prime \prime} \cdot \frac{1}{2} \frac{\partial f}{\partial z^{\prime \prime}} \equiv 0 \quad(\bmod . \delta)
$$

und folglich

$$
\frac{1}{2} \frac{\partial f}{\partial\left(q^{\prime} q_{1}^{\prime \prime}-q^{\prime \prime} q_{1}^{\prime}\right)} \equiv 0, \quad \frac{1}{2} \frac{\partial f}{\partial\left(q^{\prime \prime} q_{1}-q q_{1}^{\prime \prime}\right)} \equiv 0, \quad \frac{1}{2} \frac{\partial f}{\partial\left(q q_{1}^{\prime}-q^{\prime} q_{1}\right)} \equiv 0 \quad(\bmod . \delta) .
$$

Hiernach lassen die Gleichungen (12.) zunächst erkennen, dass $p_{2}, q_{2}, q_{2}^{\prime}, q_{2}^{\prime \prime}$ den Theiler $\delta$ gemeinsam haben.

Sie haben aber auch, wenn $h=1$ ist, den gemeinsamen Theiler 2. Um dies zu beweisen, müssen verschiedene Fälle unterschieden werden, jenachdem $q, q^{\prime}, q^{\prime \prime}$ den Grössen $q_{1}, q_{1}^{\prime}, q_{1}^{\prime \prime}$ resp. gleichartig oder ungleichartig sind. Da in allen der Gang des Beweises derselbe bleibt, beschränken wir uns hier auf die genauere Betrachtung desjenigen, in welchem $q_{1} \equiv q, q_{1}^{\prime} \equiv q^{\prime}$, $q_{1}^{\prime \prime} \equiv q^{\prime \prime}+1(\bmod .2)$ sind. Da

$$
p^{2}-F\left(q, q^{\prime}, q^{\prime \prime}\right)=2 \delta, \quad p_{1}^{2}-F\left(q_{1}, q_{1}^{\prime}, q_{1}^{\prime \prime}\right)=2 \delta,
$$

findet man

$$
\begin{aligned}
& p_{1}^{2}-F\left(q_{1}, q_{1}^{\prime}, q_{1}^{\prime \prime}\right) \equiv p_{1}^{2}-\left(q^{2}+q^{\prime 2}+q^{\prime 2}\right)-1 \equiv 0, \\
& p^{2}-F\left(q, q^{\prime}, q^{\prime \prime}\right) \equiv p^{2}-\left(q^{2}+q^{\prime 2}+q^{\prime \prime 2}\right) \equiv 0,
\end{aligned}
$$

also, $p_{1} \equiv p+1(\bmod .2)$. Daraus folgt $q_{2} \equiv q_{2}^{\prime} \equiv q+q^{\prime}, q_{2}^{\prime \prime} \equiv p+q^{\prime \prime}(\bmod .2)$.

Ist nun $p$ gerade, also $F\left(q, q^{\prime}, q^{\prime \prime}\right)$ oder $A q^{2}+A^{\prime} q^{\prime 2}+A^{\prime \prime} q^{\prime \prime 2} \equiv 2$ (mod.4), so ist $p_{1}$ ungerade, also $F\left(q_{1}, q_{1}^{\prime}, q_{1}^{\prime \prime}\right)$ oder $A q_{1}^{2}+A^{\prime} q_{1}^{\prime 2}+A^{\prime \prime} q_{1}^{\prime \prime 2} \equiv 3(\bmod .4)$, was mit Bezug auf die Voraussetzungen auch so geschrieben werden kann: 
$A^{\prime} q^{2}+A^{\prime} q^{\prime 2}+A^{\prime \prime} q^{\prime \prime 2}+A^{\prime \prime}\left(2 q^{\prime \prime}+1\right) \equiv 3 \operatorname{oder} A^{\prime \prime}\left(2 q^{\prime \prime}+1\right) \equiv 1(\bmod .4)$. Wäre $A^{\prime \prime} \equiv 3$ (mod.4), so müsste $q^{\prime \prime}$ ungerade sein, woraus $A q^{2}+A^{\prime} q^{\prime 2} \equiv 3(\bmod 4)$ folgen würde; dies ist nur so möglich, dass noch eine $\operatorname{der}$ Grössen $A, A^{\prime}$ von $\operatorname{der}$ Form $4 n+3$ ist, gegen unsere Voraussetzungen. Man müsste also $A^{\prime \prime} \equiv 1$ (mod. 4) voraussetzen, wo dann $q^{\prime \prime}$ und folglich auch $q_{2}^{\prime \prime}$ gerade würde; ferner muss $A q^{2}+A^{\prime} q^{\prime 2} \equiv 2(\bmod .4)$ sein, was nur dann möglich, wenn $q, q^{\prime}$ ungerade, folglich $q_{2}$ und $q_{2}^{\prime}$ gerade sind. Da so $q_{2}, q_{2}^{\prime}, q_{2}^{\prime \prime}$ gerade gefunden sind, muss es auch $p_{2}$ sein. Wird aber $p$ ungerade vorausgesetzt, so ist $p_{1}$ gerade, und man gelangt zu denselben Resultaten, wenn man von der zweiten Gleichung ausgeht.

Wir haben im Vorigen bewiesen, dass $p_{2}, q_{2}, q_{2}^{\prime}, q_{2}^{\prime \prime}$ durch $2^{h} . \delta$ theilbar sind. Schreibt man daher in den Gleichungen (12.) für jene Grössen resp. $2^{h} \delta . t, 2^{h} \delta . u, 2^{h} \delta^{\prime} \cdot u^{\prime}, 2^{h} \delta . u^{\prime \prime}$, so bestimmen diese Gleichungen eine Auflösung der Gleichung (10.) in ganzen Zahlen $t, u, u^{\prime}, u^{\prime \prime}$.

Dritten s erhält man aus den Gleichungen (12.) umgekehrt die folgenden :

$$
\begin{gathered}
p_{1}=t p+u \cdot \frac{1}{2} \frac{\partial F}{\partial q}+u^{\prime} \cdot \frac{1}{2} \frac{\partial F}{\partial q^{\prime}}+u^{\prime \prime} \cdot \frac{1}{2} \frac{\partial F}{\partial q^{\prime \prime}}, \\
q_{1}=t q+p u+\frac{1}{2} \frac{\partial f}{\partial\left(u^{\prime} q^{\prime \prime}-u^{\prime \prime} q^{\prime}\right)}, \quad q_{1}^{\prime}=t q^{\prime}+p u^{\prime}+\frac{1}{2} \frac{\partial f}{\partial\left(u^{\prime \prime} q-u q^{\prime \prime}\right)}, \\
q_{1}^{\prime \prime}=t q^{\prime \prime}+p u^{\prime \prime}+\frac{1}{2} \frac{\partial f}{\partial\left(u q^{\prime}-u^{\prime} q\right)},
\end{gathered}
$$

welche zeigen, dass die Auflösung $p_{1}, q_{1}, q_{1}^{\prime}, q_{1}^{\prime \prime} \operatorname{der}$ Gleichung (3.) aus der Zusammensetzung der Auflösung $p, q, q^{\prime}, q^{\prime \prime}$ derselben Gleichung mit der Auflösung $t, u, u^{\prime}, u^{\prime \prime}$ der Gleichung (10.) nach der Formel

$$
p_{1}^{2}-F\left(q_{1}, q_{1}^{\prime}, q_{1}^{\prime \prime}\right)=\left[t^{2}-F\left(u, u^{\prime}, u^{\prime \prime}\right)\right] \cdot\left[p^{2}-F\left(q, q^{\prime}, q^{\prime \prime}\right)\right]
$$

erhalten werden kann.

Somit ist der Satz vollständig bewiesen. -

Der zweite Punkt des Beweises, den wir rein arithmetisch gegeben haben, lässt sich auch aus den Transformationsgleichungen folgendermassen ableiten. Man bezeichne mit $S$ die, durch die Gleichungen (1.) definirte Transformation der Form $f$ in sich selbst, während $p, q, q^{\prime}, q^{\prime \prime}$ eine bestimmte Auflösung der Gleichung (3.) auf ein gegebenes Werthsystem $h, \delta$ bezüglich bezeichnen. Ist $S_{1}$ die ähnliche durch eine Lösung $p_{1}, q_{1}, q_{1}^{\prime}, q_{1}^{\prime \prime}$ derselben Gleichung bestimmte Transformation, so erhält man durch successive Auwendung von $S$ und $S_{1}$ eine neue Transformation von $f$ in sich selbst; und zwar zeigt eine genauere Untersuchung, dass man die zusammengesetzte Transformation aus den Gleichungen (1.) erhält, indem man darin $p, q, q^{\prime}, q^{\prime \prime}$ 
durch diejenigen Grössen $p_{2}, q, q_{2}^{\prime}, q_{2}^{\prime \prime}$ ersetzt, die nach der Regel der No.2 durch Zusammensetzung der Ausdrücke

$$
p^{2}-F\left(q, q^{\prime}, q^{\prime \prime}\right) \text { und } p_{1}^{2}-F\left(q_{1}, q_{1}^{\prime}, q^{\prime \prime}\right) \quad \text { in } p_{2}^{2}-\boldsymbol{F}\left(q_{2}, q_{2}^{\prime}, q^{\prime \prime}\right)
$$

erhalten werden. Den grössten gemeinsamen Theiler, welchen $p_{2}, q_{2}, q_{2}^{\prime}, q_{2}^{\prime \prime}$ etwa haben, bezeichnen wir mit $d$, und setzen $p_{2}=d t, q_{2}=d u, q_{2}^{\prime}=d u^{\prime}, q_{2}^{\prime \prime}=d u^{\prime \prime}$. Dividirt man die Gleichungen (1.) durch $d^{2}$, so erhält man ein ähnliches System von Gleichungen, in welchen $p, q, q^{\prime}, q^{\prime \prime}$ durch $t, u, u^{\prime}, u^{\prime \prime}$ ersetzt sind, und welche die zusammengesetzte Transformation $S . S_{1}$ darstellen. Der Ausdruck $t^{2}-F\left(u, u^{\prime}, u^{\prime \prime}\right)$, dessen Werth gleich $\frac{2^{2 h} \cdot \delta^{2}}{d^{2}}$ ist, muss also von der Form $2^{h^{\prime}} . \delta^{\prime}$ sein, wo $h^{\prime}=0$ oder $1, \delta^{\prime}$ ein Theiler von $A$ ist, was nur möglich ist, wenn $h^{\prime}=0, \delta^{\prime}=1$. Der grösste gemeinsame Theiler $d$ ist also gleich $2^{h} . \delta$, und $t, u, u^{\prime}, u^{\prime \prime}$ eine ganzzahlige Auflösung der Gleichung (10.).

4. Als Resultat dieser Untersuchung können wir folgenden Satz aussprechen:

Bezeichnen wir mit $S_{1}, S_{1}, S_{2}, \ldots$ die, stets in endlicher Anzahl vorhandenen singulären Transformationen, welche aus den Gleichungen (1.) entstehen, indem man darin für $p, q, q^{\prime}, q^{\prime \prime}$ je eine Auflösung der Gleichungen von der Form $p^{2}-F\left(q, q^{\prime}, q^{\prime \prime}\right)=2^{h} . \delta$ setzt, deren $p$ durch $\delta$ theilbar ist, mit $T$ aber die Transformationen, welche den sämmtlichen Lösungen $p, q, q^{\prime}, q^{\prime \prime}$ der Gleichung $p^{2}-F\left(q, q^{\prime}, q^{\prime \prime}\right)=1$ entsprechen, so zerfallen alle möglichen ganzzahligen Transformationen in die Gruppen:

$$
\text { T. } S_{1)}, \quad T . S_{1}, \quad T . S_{2}, \quad \ldots
$$

Nehmen wir als Beispiel die Form $x^{2}+x^{\prime 2}-x^{\prime 2}$, deren Adjungirte ihr selbst gleich ist; da ihre Determinante gleich Eins ist, zerfällt die Gleichung (3.) hier in die vier folgenden:
(13".) $p^{2}-q^{2}-q^{\prime 2}+q^{\prime \prime 2}=1$,
(13 $\left.3^{b}\right) \quad p^{2}-q^{2}-q^{\prime 2}+q^{\prime \prime 2}=2$,
(13 $\left.3^{c}\right) p^{2}-q^{2}-q^{\prime 2}+q^{\prime \prime 2}=-1$,
$\left(13^{d}\right.$.) $p^{2}-q^{2}-q^{\prime 2}+q^{\prime 2}=-2$.

Man erbält daher alle Transformationen der Form $x^{2}+x^{\prime 2}-x^{\prime 2}$ in sich selbst mittelst folgender Formeln

(14.)

$$
\left\{\begin{array}{c}
\left(p^{2}-q^{2}-q^{\prime 2}+q^{\prime 2}\right) X \\
=\left(p^{2}-q^{2}+q^{\prime 2}-q^{\prime \prime 2}\right) x-2\left(p q^{\prime \prime}+q q^{\prime}\right) x^{\prime}-2\left(p q^{\prime}+q q^{\prime \prime}\right) x^{\prime \prime} \\
\left(p^{2}-q^{2}-q^{\prime 2}+q^{\prime 2}\right) X^{\prime} \\
=2\left(p q^{\prime \prime}-q q^{\prime}\right) x+\left(p^{2}+q^{2}-q^{\prime 2}-q^{\prime \prime 2}\right) x^{\prime}+2\left(p q-q^{\prime} q^{\prime \prime}\right) x^{\prime \prime} \\
\left(p^{2}-q^{2}-q^{\prime 2}+q^{\prime \prime 2}\right) X^{\prime \prime} \\
=-2\left(p q^{\prime}-q q^{\prime \prime}\right) x+2\left(p q+q^{\prime} q^{\prime \prime}\right) x^{\prime}+\left(p^{2}+q^{2}+q^{\prime 2}+q^{\prime \prime 2}\right) x^{\prime \prime}
\end{array}\right.
$$


304 Bachmann, zur Transformation der ternären quadratischen Formen.

wenn darin für $p, q, q^{\prime}, q^{\prime \prime}$ alle ganzzahligen Auflösungen der Gleichungen (13.) substituirt werden. Der Auflösung $p=1, q=0, q^{\prime}=0, q^{\prime \prime}=-1$ der Gleichung $\left(13^{b}.\right)$ entspricht die singuläre Transformation $\left.S=\begin{array}{r}0,1,0 \\ -1,0,0 \\ 0,0,1\end{array}\right)$. Jede andere, einer Auflösung dieser Gleichung entsprechende Transformation muss also erhalten werden können, indem man $S$ mil einer der Gleichung $\left(13^{a}\right.$.) entsprechenden Transformation zusammensetzt. So ergiebt sich in der That

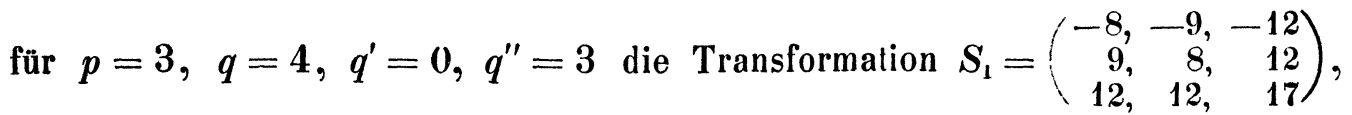
und es ist $S_{1}=T . S$, worin $T$ die, der Auflösung $p=0, q=2, q^{\prime}=-2, q^{\prime \prime}=3$ der Gleichung $\left(13^{a}.\right)$ entsprechende Transformation $\left(\begin{array}{rr}-9,+8, & -12 \\ 8,-9, & 12 \\ 12,-12, & 17\end{array}\right)$ bezeichnet.

Bemerken wir ferner, dass aus einer Auflösung $p=p_{0}, q=q_{0}, q^{\prime}=q_{0}^{\prime}$, $q^{\prime \prime}=q_{0}^{\prime \prime}$ der Gleichungen $\left(13^{c}\right.$.) und $\left(13^{d}\right.$.) sofort die Auflösung $p=q_{0}, q=p_{0}$, $q^{\prime}=q_{u}^{\prime \prime}, q^{\prime \prime}=q_{v}^{\prime}$ der Gleichungen $\left(13^{a}.\right)$ und $\left(13^{b}.\right)$ resp. sich ergiebt, so finden wir leicht, dass die den Gleichungen $\left(13^{c}\right.$.) und $\left(13^{d}\right.$.) entsprechenden Transformationen resp. aus den zu den Gleichungen $\left(13^{a}\right.$.) und $\left(13^{b}\right.$.) gehörigen erhalten werden, indem man $x^{\prime}, x^{\prime \prime}$ in $-x^{\prime},-x^{\prime \prime}$ verwandelt, d. h. mittelst der Transformation $S_{0}=\left(\begin{array}{rrr}1, & 0, & 0 \\ 0, & -1, & 0 \\ 0, & 0, & -1\end{array}\right)$. Hiernach erhält man alle Transformationen der Form $x^{2}+x^{\prime 2}-x^{\prime \prime 2}$ in sich selbst mittelst der Formeln

$$
\text { T, T.S. }
$$

Betrachtet man aber die der zweiten und vierten dieser Formeln entsprechenden als unwesentlich von den andern verschieden, so kann man sagen: Man erhält alle Transformationen der Form $x^{2}+x^{\prime 2}-x^{\prime \prime 2}$ in sich selbst mittelst der Gleichungen (14.), wenn darin für. $p, q, q^{\prime}, q^{\prime \prime}$ alle ganzzahligen Auflösungen der Gleichungen $\left(13^{a}.\right)$ und $\left(13^{h}.\right)$ substituirt werden. In dieser Form ist unser Salz, wie leicht zu sehen, mit dem in Gauss' op. II pag. 311 befindlichen identisch; in der That, von den dort unterschiedenen beiden Arten, die Gleichung $\alpha \delta-\beta \gamma=1 \mathrm{zu}$ befriedigen, entspricht die erstere der Gleichung $\left(13^{a}.\right)$, die zweite der Gleichung $\left(13^{b}.\right)$.

Breslau, im November 1869. 